NBER WORKING PAPER SERIES

\title{
BELIEF FLIPPING IN A DYNAMIC MODEL OF STATISTICAL DISCRIMINATION
}

\author{
Roland G. Fryer, Jr. \\ Working Paper 12174 \\ http://www.nber.org/papers/w12174 \\ NATIONAL BUREAU OF ECONOMIC RESEARCH \\ 1050 Massachusetts Avenue \\ Cambridge, MA 02138 \\ April 2006
}

I would like to give special thanks to Tomas J. Sjööströöm and Glenn C. Loury for continued support and advice on this project. I would also like to thank Gary Becker, Kalyan Chatterjee, Kim-Sau Chung, Hanming Fang, Vijay Krishna, Jim Jordan, Xue Jun, Steven Levitt, Brian McCannon, Andrea Moro, Roger Myerson, Peter Norman, Tor Winston, and seminar participants at the Universities of Chicago, Virginia, and Pennsylvania State. Please direct all correspondence to the author at: Harvard Society of Fellows and NBER; Littauer Center; 1875 Cambridge Street; Cambridge, MA 02138. E-mail: rfryer@ fas.harvard.edu. The usual caveat applies.

The views expressed herein are those of the author(s) and do not necessarily reflect the views of the National Bureau of Economic Research.

(C2006 by Roland G. Fryer, Jr. All rights reserved. Short sections of text, not to exceed two paragraphs, may be quoted without explicit permission provided that full credit, including $\odot$ notice, is given to the source. 
Belief Flipping in a Dynamic Model of Statistical Discrimination

Roland G. Fryer, Jr.

NBER Working Paper No. 12174

April 2006

JEL No. J7

\begin{abstract}
The literature on statistical discrimination shows that ex-ante identical groups may be differentially treated in discriminatory equilibria. This paper constructs a dynamic model of statistical discrimination and explores what happens to the individuals who nonetheless overcome the initial discrimination. If an employer discriminates against a group of workers in her initial hiring, she may actually favor the successful members of that group when she promotes from within the firm. The worker's welfare implications (i.e. who benefits from an employer's discriminatory hiring practices) are unclear. Even though agents face discrimination initially, some may be better off because of it.
\end{abstract}

\author{
Roland G. Fryer, Jr. \\ Department of Economics \\ Harvard University \\ Littauer Center, M-7 \\ Cambridge, MA 02138 \\ and NBER \\ rfryer@fas.harvard.edu
}




\section{Introduction}

The theoretical literature on statistical discrimination shares a common theme: equilibria exist in which a group is discriminated against relative to another, even when groups are ex-ante identical and employers have no psychic preference for either group. The question that has been hitherto ignored, within these models, is what happens to the agents who overcome the initial adversity in hiring and are assigned to a job within a firm. This paper addresses this question by formalizing the following simple intuition. Suppose an employer has negative stereotypes about a particular group (group A, say) and discriminates against them in her initial hiring practices, relative to another group (group B) for whom she has more positive stereotypes. Then, conditional on being hired, group A workers within the firm are relatively more talented than group B workers. This result arises because group A workers in the firm were held to a more exacting standard in initial hiring, relative to B's. Employers may take this into account in their favor when they promote from within the firm. Thus, although A's are subjected to initial adversity, once hired, they may be more likely to be promoted. ${ }^{1}$ I refer to this as "belief-flipping" - being pessimistic about a group in general, but optimistic about the successful members of that group.

The main result in this paper is a sufficient condition for "belief flipping" to arise in a dynamic equilibrium of a simple two-stage job assignment game. Whether or not belief-flipping obtains is driven by two effects: a talent effect and an investment effect. In standard (one-stage) models of statistical discrimination, when an employer changes her hiring standards, this influences the worker's investment behavior (investment effect). In a dynamic model, an employer's asymmetric initial hiring standards also induce an endogenous talent effect in later stages of the game. The talent effect is always positive; higher initial standards imply a more talented pool of workers in the firm. The investment effect, however, may be positive or negative because there may be continued discrimination in the promotion stage. If the investment effect is positive, belief-flipping will arise. If the investment effect is negative, then the magnitude of the talent effect must outweigh that of the investment effect. Careful attention to the details will highlight the following "rule of thumb:" if a worker's promotion stage wages and the employer's profit margin on offered wages take on intermediate values, belief-flipping can occur.

\footnotetext{
${ }^{1}$ Of course, this does not imply that the number of A's that are promoted will be more than the number of B's. Or, that ex-ante expected welfare of A's will be greater than B's.
} 
This result may seem innocuous. Technically, beliefs can flip in one-stage statistical discrimination models if the game were to be trivially repeated twice, and group B, for example, was discriminated against the first time the game was played relative to group A, and the second time group A was discriminated against relative to group B. This type of crude argument, however, is (1) not particularly interesting, given there is no link between the stages (i.e., employers cannot make inferences about workers in later stages of their career, as a function of their previous play) and (2) implicitly relies on an unspecified equilibrium selection mechanism that for some (ad hoc) reason chooses to discriminate against Bs the first time the game is played and discriminate against As the second time the game is played. In the model presented here, there is an explicit link between the two periods that allows the employer to discern group specific characteristics about the workers over time.

When belief-flipping occurs, who benefits from the employer's discriminatory hiring practices is unclear. The ex-ante expected payoff of the group who faces the initial discrimination is always lower than the group who does not face this discrimination. Ex-post, however, workers who are hired despite the employer's animus towards their group benefit from the initial adversity. More succinctly, B's would prefer to be A's before the initial hiring process, but conditional upon being hired, they are happy to be B's.

The seminal contributions to the statistical discrimination literature consist of Phelps (1972) and Arrow (1973). Phelps (1972) assumes that the signal minorities emit is nosier, and therefore, employers (rationally) discriminate against them in equilibrium. Arrow (1973) argues that employers can (rationally) discriminate against a group, even when they are ex-ante identical, and the employer herself does not have taste-based group prejudice. He notes that when some employee characteristics are endogenous, an employer's a priori beliefs can be self-fulfilling. Two important contributions in this literature are Coate and Loury (1993) and Moro and Norman (2004). Coate and Loury (1993) provide a simple model of employer-worker interaction that captures the ideas expressed in Arrow (1973), and then use the model to evaluate whether affirmative action policies will eliminate statistical discrimination. Moro and Norman (2004) extend the model of Coate and Loury (1993) to allow for endogenous wages; analyzing affirmative action in a competitive market. The current model serves as a theoretical extension of Arrow (1973), that encompasses some aspects of Coate and Loury (1993). To make the discussion reader-friendly, I adopt much of the language, 
notation, and style of the original Coate and Loury (1993) paper.

\section{A Dynamic Model of Statistical Discrimination}

\section{A. The Basic Building Blocks}

Let there be one agent referred to as a worker and one agent referred to as an employer. Nature moves first and assigns a type to the worker. This type, denoted $c^{j}$, depicts the worker's cost of investing in each stage $j, j \in\{h, p\}$, where $c^{j} \sim U[0,1]$, and $c^{h}=c^{p}=c^{2}$ After observing his cost, the worker makes a dichotomous hiring-stage investment decision, choosing to become either "qualified" or "unqualified," with no in-between. Then, nature distributes a signal $\theta^{h} \in$ \{pass, fail, unclear\} regarding the worker's investment decision, where $\theta^{h} \in\{$ pass, unclear $\}$ if the worker invested and $\theta^{h} \in\{$ fail, unclear $\}$ if not.

Next, the employer observes $\theta^{h}$ and makes a deterministic hiring decision: hire or reject. If the worker is rejected, he exits the game and takes no further action. If the worker is hired, he proceeds to the promotion stage. Between the hiring and promotion stages, the employer observes the worker's hiring-stage investment decision. If they are not qualified, and cost the firm profits, an employer will not promote the worker. Thus, workers who do not invest in the hiring stage are not eligible for a promotion regardless of their promotion stage signal. To keep things tractable, those who invested in the hiring stage, but were not hired, are not eligible for re-entry in the promotion stage. $^{3}$

If the worker made the hiring-stage investment decision, then in the promotion stage he makes another investment decision, choosing whether or not to be qualified for the promotion opportunity. Then, nature distributes a noisy signal to the employer regarding the worker's investment decision, $\theta^{p} \in\{$ pass, fail, unclear $\}$, where (as before) $\theta^{p} \in\{$ pass, unclear $\}$ if the worker is qualified and $\theta^{p} \in\{$ fail, unclear $\}$ if the worker is unqualified. After observing the signal, the employer makes a promotion decision, deciding whether to promote the worker or leave him in the initial hiring stage position.

\footnotetext{
${ }^{2}$ Ideally, one might want the cost to be correlated, but not perfectly. All the results go through if the correlation between the costs in each stage is sufficiently high.

${ }^{3}$ These assumptions can be justified if some on-the-job-training is required for the promotion. Thus, those workers who were passed over in the initial hiring stage, or did not invest in the initial hiring stage, do not have the necessary skills for the promotion.
} 


\section{B. Payoffs}

If the worker is hired, he receives a fixed payoff of $1-c$ if he chose to invest in the hiring stage and is normalized to 1 if not. The employer's net payoff for hiring a worker is normalized to 0 if the worker is qualified and $-1<0$ if the worker is unqualified, given wages are paid while production occurs. The payoff to the employer for rejecting the worker is zero.

In the promotion stage, the worker receives a payoff of $\lambda-c$ if he is promoted and qualified for the promotion, and $\lambda$ if he is promoted and not qualified, $\lambda>1$. If the worker is not promoted, but invested in the hiring stage, then he stays in the same job and receives a payoff of 1 in the promotion stage. The employer receives a payoff of $\alpha-\lambda \geq 0$ if she promotes a worker who is qualified in the promotion stage, and $-\lambda$ if she promotes an unqualified worker. If the employer chooses not to promote a worker who was qualified in the hiring stage, she again receives a payoff of 0 . If she hires an unqualified worker in the hiring stage, and thus receives negative payoff, then she receives a payoff of zero for the promotion stage. The final payoff to each agent is the sum of the payoffs in each period with no discounting.

\section{Strategies}

Without loss of generality, the worker's strategy can be represented by cut-off points, $\left(c^{h *}, c^{p *}\right)$, such that the worker will invest in the hiring stage if $c \leq c^{h *}$, and conditional on being hired and making the investment in the hiring stage, invests in the promotion stage if $c \leq c^{p *}$, where $\left(c^{h *}, c^{p *}\right) \in[0,1]^{2}$. A strategy for the employer is an assignment decision in the hiring and promotion stages. $^{4}$

When optimizing, an employer will hire (resp. promote) a worker if they observe a passing signal and reject a worker if they observe a failing signal. When the signal is ambiguous, the hiring/promotion decision is more complicated. Let $\rho_{q}$ (resp. $\rho_{u}$ ) denote the probability that a worker receives an unclear test score when he is qualified (resp. unqualified), and let $\mu^{j} \in[0,1]$

\footnotetext{
${ }^{4}$ Formally, the worker's strategy consists of a pair of functions (investment decisions) $I^{h}:[0,1] \rightarrow\{0,1\}$, where $I^{h}$ is known as the worker's hiring-stage investment function, and $I^{p}:\{0,1\} \times[0,1] \rightarrow\{0,1\}$ where $I^{p}$ is known as the worker's promotion-stage investment function. The employer's strategies also consist of a pair of functions (hiring/promotion decisions) $A^{h}:$ pass, fail, unclear $\} \rightarrow\{0,1\}$, where $A^{h}$ is known as the employer's initial hiring function, and $A^{p}: \theta^{h} \in\{0,1\} \times\{$ pass, fail, unclear $\} \rightarrow\{0,1\}$, where $A^{p}$ is known as the employer's promotion function.
} 
denote the probability that the employer hires (resp. promotes) a worker in stage $j$, when she observes an unclear test result. ${ }^{5}$ An employer is said to be "liberal" ( $\left.\mu^{j}=1\right)$ towards a group, in stage $j$, if she gives them the "benefit of the doubt" and conservative $\left(\mu^{j}=0\right)$, in stage $j$, if not.

\section{Expected Payoffs}

Let $\pi^{j} \in[0,1]$ denote the employer's prior belief that the worker is qualified for stage $j \in\{h, p\}$. Expected payoffs for the employer are functions of her beliefs and the signal she observes. Specifically, given $\pi^{j}$ and observed signal $\theta^{j}$, she formulates a posterior probability, denoted $\Psi\left(\pi^{j}, \theta^{j}\right)$ (using Bayes's rule), that the worker is qualified in stage $j, j \in\{h, p\}$.

In particular, $\Psi\left(\pi^{j}\right.$, pass $)=1, \Psi\left(\pi^{j}\right.$, fail $)=0$, and $\Psi\left(\pi^{j}\right.$, unclear $)=\frac{\pi^{j} \rho_{q}}{\pi^{j} \rho_{q}+\left(1-\pi^{j}\right) \rho_{u}}$. Given these posterior probabilities, the employer's expected payoff for promoting a worker with an unclear signal is

$$
\Psi\left(\pi^{p}, \text { unclear }\right)(\alpha-\lambda)-\left[1-\Psi\left(\pi^{p}, \text { unclear }\right)\right] \lambda
$$

Let $V\left(\pi^{p}\right)$ denote the employer's expected value of participation in the promotion stage, where

$$
V\left(\pi^{p}\right) \equiv \pi^{p}\left(1-\rho_{q}\right)(\alpha-\lambda)+\mu^{p}\left[\rho_{q} \pi^{p}(\alpha-\lambda)-\rho_{u}\left(1-\pi^{p}\right) \lambda\right]
$$

The employer's expected payoff for hiring a worker who emits an unclear signal is then

$$
\Psi\left(\pi^{h}, \text { unclear }\right) V\left(\pi^{p}\right)-\left[1-\Psi\left(\pi^{h}, \text { unclear }\right)\right]
$$

Recall, the expected payoff for rejecting a worker in the hiring stage is normalized to zero.

The worker's expected payoff is a function of the hiring and promotion thresholds set by the employer, the probability that he is promoted if he emits an unclear signal $\left(\mu^{p}\right)$, the wages paid, and his cost. Let $R_{\mu^{p}}(c)$ denote the worker's expected return in the promotion stage, where

$$
R_{\mu^{p}}(c) \equiv \max \left\{\rho _ { q } \left[\mu^{p} \lambda+\underset{\text { invest }}{\left.\left.\mu^{p}\right)\right]+}\left(1-\rho_{q}\right) \lambda-c ; \rho_{u}\left[\mu^{p} \lambda+\underset{\text { do not invest }}{\left.\left(1-\mu^{p}\right)\right]+}\left(1-\rho_{u}\right)\right\}\right.\right.
$$

To ensure that the incentive to invest in the promotion stage is larger when an employer is liberal (relative to a conservative employer), we assume $\rho_{q}>\rho_{u}$.

\footnotetext{
${ }^{5}$ Allowing the precision of the testing technology to differ in the hiring and promotion stages is a straightforward extension. All derivations with this extension are available from the author upon request.
} 
In the hiring stage, expected payoffs depend on the worker's expectation of facing liberal/conservative employment standards in the hiring and promotion stages, the wages paid in each stage, and his investment costs. The worker's ex-ante expected payoffs can be written succinctly as

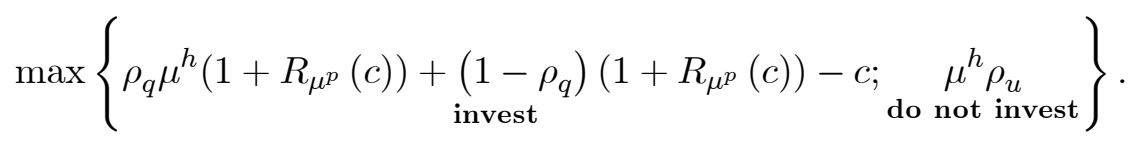

\section{E. Equilibrium}

In multi-stage models, information in the first stage influences behavior in the subsequent stages. In this model, the first stage only influences the second stage through $c^{h *}$. Therefore, once we find $c^{h *}$, this model is similar to Coate and Loury (1993) with a simple adaptation of payoffs for the employer and the worker to account for three job levels instead of two, and the possibility of endogenous asymmetric cost distributions in the promotion stage.

In any equilibrium, $\mu^{j}$ will depend on the employer's beliefs in each stage $j\left(\pi^{j}\right)$. I signify this by writing $\mu^{h *}\left(\pi^{h}, \pi^{p}\right)$ and $\mu^{p *}\left(\pi^{h}, \pi^{p}\right)$, which represent the equilibrium probabilities that a worker gets hired or promoted when he emits an unclear signal. Let $c^{h *}\left(\mu^{h}, \mu^{p}\right)$ and $c^{p *}\left(\mu^{p}\right)$ represent the worker's equilibrium cost cut-off points as a function of the employer he expects to face and define mappings $\Phi^{h}:[0,1]^{2} \rightrightarrows[0,1]$ and $\Phi^{p}:[0,1]^{2} \rightrightarrows[0,1]$ such that

$$
\begin{aligned}
\Phi^{h}\left(\pi^{h}, \pi^{p}\right) & \equiv c^{h *}\left(\mu^{h *}\left(\pi^{h}, \pi^{p}\right), \mu^{p *}\left(\pi^{h}, \pi^{p}\right)\right) \\
\Phi^{p}\left(\pi^{h}, \pi^{p}\right) & \equiv \frac{c^{p *}\left(\mu^{p *}\left(\pi^{h}, \pi^{p}\right)\right)}{c^{h *}\left(\mu^{h *}\left(\pi^{h}, \pi^{p}\right), \mu^{p *}\left(\pi^{h}, \pi^{p}\right)\right)} .
\end{aligned}
$$

Definition 1 For a given $\pi^{h *}$, an equilibrium in the promotion stage is a belief, $\pi^{p *}$, satisfying $\pi^{p *}=\Phi^{p}\left(\pi^{h *}, \pi^{p *}\right)$.

This definition of equilibrium implies that an employer's negative (resp. positive) beliefs are confirmed in equilibrium vis-à-vis a self confirming feedback loop. Suppose that the employer begins with beliefs $\pi^{h *}$ and $\pi^{p *}$ ( $\pi^{h *}$ fixed for this thought experiment). These beliefs induce the employer to promote workers with unclear test results with probability $\mu^{p}\left(\pi^{h}, \pi^{p}\right)$. Expecting this, the worker calculates $c^{p *}\left(\mu^{p}\right)$ and invests if and only if his cost are less than the cut-off. To confirm the employer's promotion stage beliefs, $\pi^{p *}=\frac{c^{p *}\left(\mu^{p *}\left(\pi^{h *}, \pi^{p *}\right)\right)}{\pi^{h *}}$. It is straightforward to verify that 
the investment cost cut off in the promotion stage $\left(c^{p *}\right)$ is no more than the cost cut-off in the hiring stage $\left(c^{h *}\right)$. In addition, I make the following simplifying assumption.

\section{Assumption 1 I will focus on equilibria in which $c^{p *}<c^{h *}{ }^{6}$}

For transparency, I have concentrated thus far on a one group (or multiple groups for which the employer has identical beliefs) model in the discussion of equilibrium. When multiple equilibrium exists, there can be discriminatory treatment - one group is held to a more exacting hiring standard because of an employer's relatively pessimistic beliefs.

I begin the equilibrium characterization with the worker's equilibrium behavior. The worker will invest in the promotion stage if and only if the cost of the investment is less than the net benefit, which is comprised of two quantities: the net return from being promoted $(\lambda-1)$ and the increased probability of promotion due to investing. The worker's expectations over the latter quantity depends on how he expects to be treated if he emits an unclear signal. Formally, the net benefit of investment in the promotion stage can be derived by subtracting the first term from the second term in the max operator found in (4), disregarding $c$. The worker who is just indifferent between investing and not investing in the promotion stage has a cost $c_{\mu^{p}}^{p *}$ that is equal to the net benefit, where as a matter of notational convenience, $c_{\mu^{p}}^{p *}=c^{p *}\left(\mu^{p}\right)$.

The employer will promote the worker only if one of the following conditions hold: (1) she observes a passing score or (2) she observes an unclear score and her prior beliefs are sufficiently optimistic. The equilibrium determination of beliefs, in the promotion stage, involves a truncation of the first period distribution function. ${ }^{7}$ For $c^{h} \leq c^{h *}$, the conditional distribution function (conditional on having cost less than $c^{h *}$ ) can be written as $\frac{c^{h}}{c^{h *}}$. Without loss of generality, we can restrict our attention to workers that have $c^{h} \leq c^{h *} .8$ Finally, using the definition of equilibrium,

\footnotetext{
${ }^{6}$ This is assumption is for analytical convenience, as it allows one to simplify the workers investment criterion in the hiring stage, by ridding ourselves of the "max" terms in the worker's promotion stage expected return $\left(R_{\mu} p(c)\right)$, and does not alter any results. Further, any generalization of the testing technology that satisfies the no shifting support property will only yield equilibrium in which Assumption 1 holds. If we were not to concentrate on equilibria in which $c^{p *}<c^{h *}$, there would be two additional cases to check in Proposition 1, which are available from the author upon request.

${ }^{7}$ If the noisy test score and costs are both functions of ability, then the distribution function is more complicated.

${ }^{8}$ This is made plausible by the assumption that if the worker does not invest in the hiring stage, he cannot invest in the promotion stage.
} 
we know the employer's beliefs in the promotion stage are a function of the employment standards in both stages via the process described here.

Definition 2 An equilibrium of the game is a pair of beliefs $\left(\pi^{h *}, \pi^{p *}\right)$ satisfying $\pi^{h *}=\Phi^{h}\left(\pi^{h *}, \pi^{p *}\right)$ and $\pi^{p *}=\Phi^{p}\left(\pi^{h *}, \pi^{p *}\right)$.

In any equilibrium of the game, the worker invests in the hiring stage if and only if his cost is less than $c^{h *}$, and $c^{h *}$ must be such that the worker with this cost is just indifferent between investing and not investing in the hiring stage. The worker's hiring-stage investment criterion can be interpreted as the net benefit of investing in the hiring stage plus the expected continuation payoff of being in the promotion stage $\left(R_{\mu^{p}}(c)\right)$. When the employer is liberal (resp. conservative) in the promotion stage, I write $R_{1}(c)$ (resp. $R_{0}(c)$ ). Formally, the investment criterion in the hiring stage can be derived by subtracting the second term from the first term of the max operator in (5).

One can think of an employer as having one of four strategies: conservative-conservative (C-C) $\left[\left(\mu^{h}, \mu^{p}\right)=(0,0)\right]$, conservative-liberal (C-L) $\left[\left(\mu^{h}, \mu^{p}\right)=(0,1)\right]$, liberal-conservative (L-C) $\left[\left(\mu^{h}, \mu^{p}\right)=(1,0)\right]$, or liberal-liberal (L-L) $\left[\left(\mu^{h}, \mu^{p}\right)=(1,1)\right]$, depending on $\mu^{h}$ and $\mu^{p}$. In what follows, equilibria are characterized for these four types of employers $\left(\left(\mu^{h,}, \mu^{p}\right) \in\{0,1\}^{2}\right)$, independently. For the sake of space, I analyze employers who play C-L and L-C strategies in the main text, since this is central to my contribution. ${ }^{9}$

\section{3 "Belief-Flipping"}

Suppose there exist two workers, one black and one white say, and the employer formulates her beliefs based solely on the workers' group identity. One-stage models of statistical discrimination assume that blacks play the conservative equilibrium and whites the liberal. In a two-stage model, then, it is feasible that the black worker will play the C-L equilibrium and the white worker the L-C equilibrium. That is, an employer may believe that a random black worker in the population is less likely to be qualified, but a black worker who is successful in obtaining a job is actually more talented.

\footnotetext{
${ }^{9}$ Derivations of the sufficient conditions for C-C and L-L equilibria to exist are available from the author upon request.
} 
Consider the following thought experiment: suppose the employer believes that the white worker is more qualified in the hiring stage than the black worker. Because of these beliefs, the firm sets a liberal hiring standard for the white worker $\left(\mu^{h}=1\right)$ and a conservative standard for the black worker $\left(\mu^{p}=0\right)$. This implies that the black worker is less probable to make the initial costly investment (holding the promotion threshold fixed), which will confirm the employer's initial hiring-stage beliefs. Conditional upon being hired, however, the black worker is more talented (lower costs) on average than the white worker. The difference is represented by an upward shift in the worker's promotion-stage investment function, which is a result of being held to a more exacting standard in the initial hiring process. Thus, in the promotion stage, it seems plausible that the employer should have relatively more optimistic beliefs about the black worker, relative to the white worker. Unfortunately, however, this is not necessarily correct. If the whites play the L-L equilibrium and blacks play the C-C equilibrium, beliefs will not flip because there is continued statistical discrimination in the promotion stage. Understanding when the beliefs will or will not flip is a subtle issue. There are two competing forces: (1) because of the initial adversity, blacks are relatively more talented than whites, conditional upon being hired (talent effect); and (2) blacks may have little incentive to invest in the promotion stage if they believe that employers will continue to discriminate (since the net investment function is single peaked). The key is under what conditions will the talent effect be strong enough to overcome any possible negative stereotypes in the promotion stage.

The next several results develop conditions under which C-L and L-C equilibrium exist and establish when belief flipping occurs. All technical proofs can be found in the Appendix.

Proposition 1 There exists a set of parameters $\left\{\underline{\alpha}, \bar{\alpha}_{C L}, \lambda_{C L}\right\}$, such that if $\alpha \in\left[\underline{\alpha}, \bar{\alpha}_{C L}\right]$ and $\lambda \leq \lambda_{C L}$ a $C$-L equilibrium exists.

Proposition 2 There exists a set of parameters $\left\{\bar{\alpha}_{L C}, \lambda_{L C}\right\}$, such that if $\alpha \leq \bar{\alpha}_{L C}$ and $\lambda \leq \lambda_{L C}$ an $L$ - $C$ equilibrium exists.

Propositions 1 and 2 provide conditions under which C-L and L-C equilibrium exist; independent of each other. For a C-L equilibrium to exist, one must have intermediate values of both $\alpha$ and $\lambda$. If $\alpha$ is too large, firms receive profit (net of wages) that is high enough for them to incur more risk in promoting workers who emit unclear signals. There is little incentive for them to remain 
conservative in the promotion stage. If $\alpha$ is too small, the opposite logic holds. The final condition involves the worker's gross wages in the promotion stage, $\lambda$. If $\lambda$ is too small, few workers will find it worthwhile to invest in the promotion stage - investing at a rate too low to sustain the employers optimistic promotion stage beliefs. If $\lambda$ is too large, forward looking workers will invest in both stages in sufficient numbers to ensure that the employer is best to be liberal in both stages. The upper bound condition on $\lambda$ is implicit in the conditions on $\alpha$.

Proposition 2 provides conditions in the same spirit for L-C equilibria. For an L-C equilibrium to exist, $\alpha$ cannot be too large (provides incentives for employers to be liberal in the promotion stage) and $\lambda$ cannot be too large (workers would invest in sufficient amounts to sustain liberal employers).

Belief-flipping occurs when all the conditions above are jointly satisfied. This is the subject of our main result.

Corollary 1 There exists a set of parameters $\left\{\underline{\alpha}, \bar{\alpha}_{C L}, \bar{\alpha}_{L C}, \lambda_{C L}, \lambda_{L C}\right\}$ such that if $\alpha \in\left[\underline{\alpha}, \min \left\{\bar{\alpha}_{C L}, \bar{\alpha}_{L C}\right\}\right]$ and $\lambda \leq \min \left\{\lambda_{C L}, \lambda_{L C}\right\}$, then $a C-L$ and an $L-C$ equilibrium coexist.

The key parameters to understand belief flipping are $\lambda$ and $\alpha$; the gross return to workers of being promoted and the profit margin on offered wages. If the profit margin is too small $(\alpha \approx \lambda)$, employers gain little from promoting its workers. As such, they are less likely to take risks, causing them to be conservative in the promotion stage. Conversely, if the profit margin is too large, the opposite is true - promoting qualified workers yields a substantial return and employers prefer to be liberal in the promotion stage. Intermediate values of $\alpha$ allow the possibility that an employer may be conservative or liberal in the promotion stage.

An identical argument establishes that $\lambda$ must also take on intermediate values. If $\lambda$ is too small, workers will not invest in the promotion stage in sufficient amounts to sustain an employers optimistic beliefs. If $\lambda$ is too big, workers will have too much incentive to invest and the employer's best response will be to set liberal standards. Intermediate values on $\alpha$ and $\lambda$ leave open the possibility that both C-L and L-C equilibrium can coexist.

\section{A. An Example}

In this section, we solve a simple example of an equilibrium with belief-flipping. For the purposes of this example, let $\rho_{q}=.8, \rho_{u}=.4, \lambda=3, \alpha=3.25$. 
The timing of events is as follows. First, nature distributes the cost of investment to each worker. Observing their cost, the worker makes their investment decision. Given the parameters above, the expected payoff to the worker in the promotion stage is:

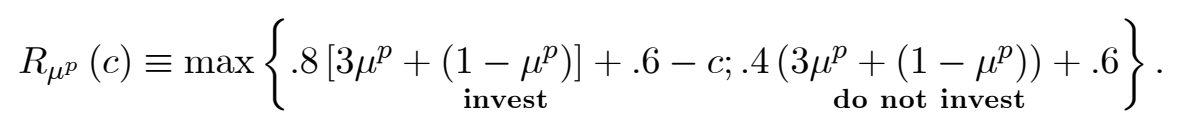

Thus, $R_{1}(c)$ and $R_{0}(c)$ can be written as:

$$
\begin{aligned}
& R_{1}(c)=\max \left\{\begin{array}{lc}
3-c ; & 1.8 \\
\text { invest } & \text { do not invest }
\end{array}\right\} \\
& R_{0}(c)=\max \left\{\begin{array}{lc}
1.4-c ; & 1 \\
\text { invest } & \text { do not invest }
\end{array}\right\}
\end{aligned}
$$

And, $c_{1}^{p *}=1.2>c_{0}^{p *}=.4$. Similarly, the hiring stage expected payoff is:

$$
\max \left\{.8 \mu^{h}\left(1+R_{\mu^{p}}(c)\right)+.2\left(1+R_{\mu^{p}}(c)\right)-c ; \quad \begin{array}{cc}
.4 \mu^{h} \\
\text { invest }
\end{array}\right\}
$$

$c_{0,1}^{h *}=.56$ and $c_{1,0}^{h *}=1.6$.

Therefore, the fraction of workers that choose to invest in both stages is:

$$
\begin{aligned}
& \pi^{h}=1 \text { and } \pi^{p}=.4 \text { in the L-C equilibrium, and } \\
& \pi^{h}=.56 \text { and } \pi^{p}=1 \text { in the C-L equilibrium. }
\end{aligned}
$$

To check that these constitute an equilibrium, we must ensure that if an employer is liberal (resp. conservative) in stage $j$, they want (resp. do not want) to hire a worker who emits an unclear signal.

Recall $V\left(\pi^{p}\right)$ denotes the employer's expected value of participation in the promotion stage, where

$$
V\left(\pi^{p}\right) \equiv \pi^{p}\left(1-\rho_{q}\right)(\alpha-\lambda)+\mu^{p}\left[\rho_{q} \pi^{p}(\alpha-\lambda)-\rho_{u}\left(1-\pi^{p}\right) \lambda\right]
$$

An employer is liberal in the hiring stage only if

$$
\Psi\left(\pi^{h}, \text { unclear }\right) V\left(\pi^{p}\right)-\left[1-\Psi\left(\pi^{h}, \text { unclear }\right)\right] \geq 0 .
$$

Inputting the parameters from the candidate L-C equilibrium, it is easy to verify that inequality (7) holds if $\alpha \geq \lambda$, which is true by definition. 
Similarly, an employer is conservative in the hiring stage only if

$$
\Psi\left(\pi^{h}, \text { unclear }\right) V\left(\pi^{p}\right)-\left[1-\Psi\left(\pi^{h}, \text { unclear }\right)\right] \leq 0 .
$$

Note that in the candidate C-L equilibrium,

$$
\Psi\left(\pi^{h}, \text { unclear }\right)=\frac{\pi^{h} \rho_{q}}{\pi^{h} \rho_{q}+\left(1-\pi^{h}\right) \rho_{u}}=\frac{.56(.8)}{.56(.8)+.44(.4)}=.7179 .
$$

Thus inequality (8) holds if:

$$
V\left(\pi^{p}\right) \leq \frac{.2820}{.7179}
$$

which can be shown by plugging the equilibrium parameters into equation 6 .

Finally, we must ensure that employers in a C-L (resp. L-C) equilibrium promote (resp. do not promote) workers with unclear promotion stage signals.

An employer's expected payoff for promoting a worker with an unclear signal is

$$
\Psi\left(\pi^{p}, \text { unclear }\right)(\alpha-\lambda)-\left[1-\Psi\left(\pi^{p}, \text { unclear }\right)\right] \lambda .
$$

An employer will be liberal only if

$$
\Psi\left(\pi^{p}, \text { unclear }\right)(\alpha-\lambda)-\left[1-\Psi\left(\pi^{p}, \text { unclear }\right)\right] \lambda \geq 0 .
$$

Plugging in the parameters of our example (i.e. $\pi^{p}=1$ ), the above inequality holds if $\alpha \geq \lambda$, which is true by definition.

An employer will be conservative in the promotion stage only if

$$
\begin{gathered}
\Psi\left(\pi^{p}, \text { unclear }\right)(\alpha-\lambda)-\left[1-\Psi\left(\pi^{p}, \text { unclear }\right)\right] \lambda \leq 0 \\
\Rightarrow .57(\alpha-\lambda)-.43 \lambda=.1425-1.29 \leq 0 .
\end{gathered}
$$

Thus, our candidate equilibrium is verified and belief-flipping occurs.

\section{B. Some Suggestive Evidence on Belief-Flipping}

There have been no direct empirical studies or simulations of multiple-stage statistical discrimination models ${ }^{10}$, although there is empirical evidence to support such models.

\footnotetext{
${ }^{10}$ There is recent work on empirical estimation of one-stage statistical discrimination models. See for example Moro (2003) and Altonji and Pierret (2001).
} 
Booth et al. (1998), using the British Household Panel Survey (BHPS), concluded that women in Britain are less likely than men to be hired, but are promoted at a higher rate. The BHPS is a nationally representative random sample of households in Britain. ${ }^{11}$ The data consists of the first five waves of the BHPS, 1991-95. Over the data period, full-time male workers had only a 9.2 percent chance of receiving a promotion each year, whereas, there was an 11.6 percent chance for full-time females. They used a simple ordered Probit model to predict the probability of promotion, based on gender. Similar to this, Groot and Maansen van den Brink (1996), using the first two waves of the BHPS, distinguish between jobs that have promotion possibilities and "dead-end jobs." They show that women are less likely to be in jobs that allow promotion, but once in a job with promotion potential, are just as likely as men to be promoted. The main difference between Groot et. al. (1996) and Booth et. al. (1998) is that the latter deletes part-time workers and includes workers who receive promotions in outside firms, to account for the probability of selection bias. ${ }^{12}$

This empirical evidence provides a brief line of argument in support of the empirical content of belief-flipping, ${ }^{13}$ but by no means is meant to be proof that the model is correct. Further empirical estimation of the model and the prevalence of belief-flipping is left for future work.

\section{Welfare Implications}

The standard interpretation of one-stage models of statistical discrimination is that multiple equilibria exist, and blacks play the bad (negative beliefs-high employment threshold) equilibrium and whites the good. In this setup, blacks receive lower expected payoffs because of the discriminatory nature of equilibria. This, however, masks the interesting welfare implications that arise in multiple stage models. Assume Proposition 1 holds, and assume blacks play the C-L equilibrium, and whites the L-C equilibrium. In this case, blacks are discriminated against relative to whites in the hiring stage, and whites are discriminated against relative to blacks in the promotion stage. Thus, contrary to the standard interpretation of one-stage models, who suffers more from the employer's discriminatory hiring practices is unclear. ${ }^{14}$ Consider six types of workers: black II (invest

\footnotetext{
${ }^{11}$ For a complete description of the data, see Taylor (1996).

${ }^{12}$ These studies and their use of the BHPS are more applicable (for testing Proposition 1) than US data because firms in Britain are not subjected to affirmative action constraints which could substantially alter equilbria.

${ }^{13}$ See also Hersch and Viscusi, 1996.

${ }^{14}$ Of course, the welfare analysis here is subject to the critique that wages are exogenous. Endogenizing wages, however, will not likely add much to the main point.
} 
in both stages), white II, black IN (invest in the hiring stage but not in the promotion stage), white IN, black N (non-investors in the hiring stage), and white N. For a given worker, his "type" will depend on the equilibrium played. It is straightforward to verify that both white and black Ns (non-investors in the hiring stage) prefer a liberal hiring standard, given their payoff is $\rho_{u}>0$ if they face a liberal hiring standard and zero if not, irrespective of the promotion stage assignment standard. If whites face an L-C employer and blacks a C-L employer, it is plausible that the black Ns will be upset, and fight for anti-discrimination policies that will enable them to have a liberal hiring standard like whites, while the white Ns are happy to play the L-C equilibrium. Interestingly, all blacks should prefer this change.

Proposition 3 The ex-ante expected payoff of any worker is higher in a $C$ - $L$ equilibrium than an $L-C$ equilibrium, whenever they exist.

This result shows that, before the hiring process begins, blacks prefer to play the white equilibrium. The intuition is transparent. For blacks to prefer the white equilibrium, the following inequality must hold:

$$
\left(1-\rho_{q}\right)\left[1+R_{1}(c)\right] \geq 1+R_{0}(c)
$$

the left hand side of which is decreasing in $\rho_{q}$. The conditions of our main proposition, however, require that $\rho_{q}$ be sufficiently large. Proposition 3 demonstrates that the two conditions cannot both be satisfied.

Ex-post, however, things are quite different.

Proposition 4 The ex-post expected payoff of a worker who invests in both stages and emits a clear signal in the hiring stage is higher in a $C$-L equilibrium than an $L$-C equilibrium, whenever they exist.

Proposition 4 shows that, while blacks initially prefer to play the white equilibrium, some are better off having endured the adversity. The fraction of workers who benefit from discrimination can be written as

$$
c_{0,1}^{h *}\left(1-\rho_{q}\right) .
$$

The fraction that continue to prefer the white equilibrium is:

$$
\left(1-c_{0,1}^{h *}\left(1-\rho_{q}\right)\right)+\rho_{q} c_{0,1}^{h *}
$$


From these conditions, one can intuit that blacks are more likely to benefit from adversity when the noise associated with the initial stage test score is relatively precise. Otherwise, they are never hired in a C-L equilibrium and the employer does not have the opportunity to update his beliefs. To get an idea of the magnitudes involved, using the parameters in the example above, roughly $11 \%$ of the blacks playing the C-L equilibrium benefit from discrimination.

\section{Concluding Remarks}

The qualitative properties of one-stage statistical discrimination models can be misleading, as they mask the rich predictions obtained in dynamic models. In particular, I have shown that in a dynamic model of statistical discrimination beliefs can flip in equilibrium (Proposition 1). In light of this, welfare implications are unclear. The ex-ante payoff of a worker is unambiguously larger under an L-C equilibrium relative to a C-L equilibrium. Ex-post, however, a non-trivial fraction of the group who was discriminated against initially benefits from the initial discrimination.

These findings are significant for several reasons. First, they are unique to dynamic environments. Second, they show that if the difference in the employer's beliefs and hiring practices are purely a result of statistical discrimination, then some agents who have been discriminated against may benefit in later stages. Specifically, for some it may be tough to get hired, but once hired, it is easy to get promoted. This suggests some strife among agents within the same group in this model, and provides a way of distinguishing statistical discrimination from other forms of discrimination.

Future work can be pursued along several dimensions. The most straightforward, perhaps, being the analysis of labor market policies such as affirmative action in a dynamic model. Multi-staged models allow one to investigate questions involving the optimal timing of affirmative action over the life-cycle. Finally, a thorough empirical understanding of the implications of belief-flipping is of particular interest. This will require detailed firm-level data on applicant pools, individuals hired, and promotions within the firm.

\section{References}

[1] Aigner, Dennis J., and Cain, Glenn G., "Statistical Theories of Discrimination in the Labor Market," Industrial and Labor Relations Review 30 (January 1977) p. 175-87.

[2] Altonji, J., and Pierret, C., "Employer Learning and Statistical Discrimination" Quarterly Journal of Economics (February 2001) 1-37. 
[3] Arrow, Kenneth J., "The Theory of Discrimination" in Discrimination in Labor Markets, Orley Ashenfelter and Albert Rees, eds. Princeton University Press, 3-33.

[4] Booth, A., Francesconi, M., Frank, J., "Glass Ceilings or Sticky Floors?" mimeo. The University of Essex. July 1999.

[5] Coate, Stephen and Loury, Glenn C. "Will Affirmative Action Eliminate Negative Stereotypes?" American Economic Review 83 (December 1993) 1220-40.

[6] Hersch, J and Viscusi, W. (1996) "Gender Differences in Promotion and Wages" Industrial Relations Vol. 35 (4) p. 461-72

[7] Groot, M., Maassen van den Brink, H., "Glass Ceilings or Dead Ends: Job Promotion of Men and Women Compared" Economics Letters V. 53 (1996) p. 221-226.

[8] Moro, Andrea., "The Effect of Statistical Discrimination on Black-White Wage Differentials: Estimating a Model with Multiple Equilibria," International Economic Review (May 2003), 467-500.

[9] Moro, A., and Norman, P., "A General Equilibrium Model of Statistical Discrimination." Journal of Economic Theory, 114 (1), (January 2004), 1-30.

[10] Phelps, Edmund S., "The Statistical Theory of Racism and Sexism," American Economic Review, (September 1972) 659-61. 


\section{Appendix: Proofs}

Before proving our main results, we establish two Lemmas which greatly simplify the characterization of equilibrium.

Let $\pi_{\mu^{h}, \mu^{p}}^{j}$ denote the employer's equilibrium beliefs as a function of $\mu^{h}, \mu^{p}$, where, for example, $\pi_{0,0}^{j}$ denotes an employer's equilibrium beliefs about workers in stage $j$, when he is conservative in both stages. Similar to the promotion stage, a worker with an unclear test result in the hiring stage also gets the benefit of the doubt if the employer is sufficiently optimistic about his group. And, let $\widehat{\pi}^{j}$ denote the equilibrium belief that makes an employer indifferent between hiring/promoting and not in stage $j$, when she observes an unclear signal.

Lemma 2 In any equilibrium the following inequalities must hold for all $\left(\mu^{h}, \mu^{p}\right) \in\{0,1\}^{2}$ :

$c_{0, \mu^{p}}^{h *} \leq \widehat{\pi}_{0, \mu^{p}}^{h}$, if the employer is conservative in the hiring stage, (2) $\frac{c_{0}^{p *}}{c_{\mu^{h}, 0}^{h *}} \leq \widehat{\pi}^{p}$, if the employer is conservative in the promotion stage, (3) $c_{1, \mu^{p}}^{h *} \geq \widehat{\pi}_{1, \mu^{p}}^{h}$, if the employer is liberal in the hiring stage, and (4) $\frac{c_{1}^{p *}}{c_{\mu^{h}, 1}^{h *}} \geq \widehat{\pi}^{p}$, if the employer is liberal in the promotion stage.

Proof. Consider (1). Suppose by way of contradiction that there exists an equilibrium in which $c_{0, \mu^{p}}^{h *}>\widehat{\pi}_{0, \mu^{p}}^{h}$. By definition of $\widehat{\pi}_{0, \mu^{p}}^{h}$, if $c_{0, \mu^{p}}^{h *}>\widehat{\pi}_{0, \mu^{p}}^{h}$, then workers are investing in sufficient amounts to make it optimal for the employer to set a liberal hiring standard, which is a contradiction. Inequality (2) through inequality (4), in the lemma, are derived analogously.

This leads to the following result.

Lemma 3 In any equilibrium, one of the following inequalities must hold:

$$
\alpha \leq \lambda\left[\frac{\rho_{u}}{\rho_{q}}\left(\frac{c_{\mu^{h}, 0}^{h *}}{c_{0}^{p *}}-1\right)+1\right]
$$

if the employer is conservative in the promotion stage, or

$$
\alpha \geq \lambda\left[\frac{\rho_{u}}{\rho_{q}}\left(\frac{c_{\mu^{h}, 1}^{h *}}{c_{1}^{p *}}-1\right)+1\right]
$$

if the employer is liberal in the promotion stage, and one of the following inequalities must hold:

$$
V\left(\pi_{0, \mu^{p}}^{p}\right) \leq \frac{\rho_{u}}{\rho_{q}}\left[\frac{1}{c_{0, \mu^{p}}^{h *}}-1\right]
$$


if the employer is conservative in the hiring stage, or

$$
V\left(\pi_{1, \mu^{p}}^{p}\right) \geq \frac{\rho_{u}}{\rho_{q}}\left[\frac{1}{c_{1, \mu^{p}}^{h *}}-1\right]
$$

if the employer is liberal in the hiring stage.

Proof. From Lemma 2 we know that in the promotion stage, one of the following must be true in equilibrium, $\frac{c_{0}^{p *}}{c_{\mu^{h}, 0}^{h *}} \leq \widehat{\pi}^{p}$ or $\frac{c_{1}^{p *}}{c_{\mu^{h}, 1}^{h *}} \geq \widehat{\pi}^{p}$. Substituting in for $\widehat{\pi}^{p}$ and using the equilibrium condition $\frac{c^{p *}}{c^{h *}}=\pi^{p *}$, these inequalities can be rewritten as

$$
\pi_{\mu^{h}, 0}^{p *} \leq \frac{\lambda \rho_{u}}{\lambda \rho_{u}+\rho_{q}(\alpha-\lambda)}
$$

and

$$
\pi_{\mu^{h}, 1}^{p *} \geq \frac{\lambda \rho_{u}}{\lambda \rho_{u}+\rho_{q}(\alpha-\lambda)}
$$

respectively. Using some algebraic manipulation, one can rewrite these inequalities, respectively, as

$$
\alpha \leq \lambda\left[\left(\frac{\rho_{u}}{\rho_{q}}\right)\left(\frac{1}{\pi_{\mu^{h}, 0}^{p *}}-1\right)+1\right]
$$

and

$$
\alpha \geq \lambda\left[\left(\frac{\rho_{u}}{\rho_{q}}\right)\left(\frac{1}{\pi_{\mu^{h}, 1}^{p *}}-1\right)+1\right]
$$

Further, we know that in the hiring stage, one of the following must hold: $c_{1, \mu^{p}}^{h *} \geq \widehat{\pi}_{1, \mu^{p}}^{h}$ or $c_{0, \mu^{p}}^{h *} \leq$ $\widehat{\pi}_{0, \mu^{p}}^{h}$. Substituting in for $\widehat{\pi}_{\mu^{h}, \mu^{p}}^{h}$ yields

$$
c_{1, \mu^{p}}^{h *} \geq \frac{\rho_{u}}{\rho_{u}+\rho_{q} V\left(\pi_{1, \mu^{p}}^{p}\right)}
$$

and

$$
c_{0, \mu^{p}}^{h *} \leq \frac{\rho_{u}}{\rho_{u}+\rho_{q} V\left(\pi_{0, \mu^{p}}^{p}\right)}
$$

Finally, solving out for $V(\cdot)$ produces the desired result.

\section{Proof of Proposition 1.}

Suppose that the employer is a C-L employer, and sets $\mu^{h}=0$ and $\mu^{p}=1$. Expecting this, $c_{0,1}^{h *}=\left[1-\rho_{q}\right]\left[2+\rho_{u}(\lambda-1)\right]$ and $c_{1}^{p *}=\left[1-\rho_{u}\right](\lambda-1)$ respectively. By definition, an equilibrium exists only if

$$
\Phi^{h}\left(\pi^{h}, \pi^{p}\right)=c_{0,1}^{h *}=\left[1-\rho_{q}\right]\left[2+\rho_{u}(\lambda-1)\right]=\pi_{0,1}^{h *}
$$


and

$$
\Phi^{p}\left(\pi^{h}, \pi^{p}\right)=\frac{c_{1}^{p *}}{c_{0,1}^{h *}}=\frac{\left[1-\rho_{u}\right](\lambda-1)}{\left[1-\rho_{q}\right]\left[2+\rho_{u}(\lambda-1)\right]}=\pi_{0,1}^{p *}
$$

By Lemma 2, $\pi_{0,1}^{h *} \leq \widehat{\pi}_{0,1}^{h}$ and $\pi_{0,1}^{p *} \geq \widehat{\pi}^{p}$, which is the desired result.

Recall, the necessary and sufficient conditions for a C-L equilibrium are

$$
\begin{gathered}
\pi_{0,1}^{p *}=\frac{\left[1-\rho_{u}\right](\lambda-1)}{\left[1-\rho_{q}\right]\left[2+\rho_{u}(\lambda-1)\right]} \geq \widehat{\pi}^{p} \\
\pi_{0,1}^{h *}=\left[1-\rho_{q}\right]\left[2+\rho_{u}(\lambda-1)\right] \leq \widehat{\pi}_{0,1}^{h}
\end{gathered}
$$

Using Lemma 2 and inequality 9, we know

$$
\alpha \geq \lambda\left[\left(\frac{\rho_{u}}{\rho_{q}}\right)\left(\frac{\left[1-\rho_{q}\right]\left[2+\rho_{u}(\lambda-1)\right]}{\left[1-\rho_{u}\right](\lambda-1)}-1\right)+1\right] \equiv \underline{\alpha}
$$

Using inequality 10 and substituting in for $\widehat{\pi}_{0,1}^{h}$, and $V(\cdot)$, yields

$$
\begin{gathered}
\pi_{0,1}^{p *}(\alpha-\lambda)-\rho_{u}\left(1-\pi_{0,1}^{p *}\right) \lambda \leq\left(\frac{\rho_{u}}{\rho_{q}}\right)\left[\frac{1}{\left[1-\rho_{q}\right]\left[2+\rho_{u}(\lambda-1)\right]}-1\right] \\
\Rightarrow \alpha \leq \lambda+\left(\frac{\rho_{u}}{\rho_{q}}\right)\left[\frac{1}{\left[1-\rho_{q}\right]\left[2+\rho_{u}(\lambda-1)\right]}-1\right]\left(\frac{1}{\pi_{0,1}^{p *}}\right)+\frac{\rho_{u}\left(1-\pi_{0,1}^{p *}\right)}{\pi_{0,1}^{p *}} \lambda \equiv \bar{\alpha}_{C L}
\end{gathered}
$$

We must ensure that $\frac{1}{\left[1-\rho_{q}\right]\left[2+\rho_{u}(\lambda-1)\right]}-1 \geq 0 \Rightarrow$

$$
\lambda \leq \frac{1}{\rho_{u}}\left(\frac{1}{1-\rho_{q}}-2\right)+1 \equiv \lambda_{C L}
$$

Q.E.D.

\section{Proof of Proposition 2:}

Suppose that the employer is an L-C employer, and sets $\mu^{h}=1$ and $\mu^{p}=0$. Expecting this, the worker calculates $c_{1,0}^{h *}=2-\rho_{u}$ and $c_{0}^{p *}=\left[1-\rho_{q}\right](\lambda-1)$. By definition, an equilibrium exists only if

$$
\Phi^{h}\left(\pi^{h}, \pi^{p}\right)=c_{1,0}^{h *}=2-\rho_{u}=\pi_{1,0}^{h *}
$$

and

$$
\Phi^{p}\left(\pi^{h}, \pi^{p}\right)=\frac{c_{0}^{p *}}{c_{1,0}^{h *}}=\frac{\left[1-\rho_{u}\right](\lambda-1)}{\left[1-\rho_{q}\right]\left[2+\rho_{u}(\lambda-1)\right]}=\pi_{1,0}^{p *}
$$

By Lemma $2, \pi_{1,0}^{h *} \geq \widehat{\pi}_{1,0}^{h}$ and $\pi_{1,0}^{p *} \leq \widehat{\pi}^{p}$, which is the desired result. 
The necessary and sufficient conditions of an L-C equilibrium are:

$$
\pi_{1,0}^{p *}=\frac{\left[1-\rho_{q}\right](\lambda-1)}{2-\rho_{u}} \leq \widehat{\pi}^{p}
$$

and

$$
\pi_{1,0}^{h *}=2-\rho_{u} \geq \widehat{\pi}_{1,0}^{h}
$$

Using Lemma 3, we know

$$
\alpha \leq \lambda\left[\frac{\rho_{u}}{\rho_{q}}\left(\frac{2-\rho_{u}}{\left[1-\rho_{q}\right](\lambda-1)}-1\right)+1\right] \equiv \bar{\alpha}_{L C}
$$

However, we must ensure that $\frac{2-\rho_{u}}{\left[1-\rho_{q}\right](\lambda-1)}-1 \geq 0 \Rightarrow$

$$
\lambda \leq \frac{2-\rho_{u}}{\left[1-\rho_{q}\right]}+1 \equiv \lambda_{L C}
$$

Q.E.D.

\section{Proof of Corollary 1}

Using the definitions of $\underline{\alpha}, \bar{\alpha}_{C L}, \bar{\alpha}_{L C}, \lambda_{C L}$ and $\lambda_{L C}$ defined in Propositions 1 and 2 establishes the result. Q.E.D.

Proof of Proposition 3

Consider three types of workers: Ns (those who do not invest in the hiring stage), INs (those who invest in the hiring stage and not the promotion stage), and IIs (those who invest in both stages).

The payoff to an $\mathrm{N}$ worker under a C-L equilibrium is 0 . Under an L-C equilibrium, his payoff is $\rho_{u} \geq 0$, thus he prefers an L-C equilibrium.

The payoff to an II worker under a C-L equilibrium is:

$$
\left(1-\rho_{q}\right)\left[1+R_{1}(c)\right]=\left(1-\rho_{q}\right)[1+\lambda-c]
$$

The payoff to an II worker under an L-C equilibrium is:

$$
\left[1+R_{0}(c)\right]=\left[1+\rho_{q}+\left(1-\rho_{q}\right) \lambda-c\right]
$$

This boils down to:

$$
\left(1-\rho_{q}\right)(1-c) \leq 1-c+\rho_{q}
$$


Finally, we must show that INs prefer an L-C equilibrium to a C-L equilibrium whenever they both exist. Suppose, by way of contradiction, that INs preferred C-L equilibrium to L-C equilibrium. Then, the following inequality must hold:

$$
\begin{aligned}
& \left(1-\rho_{q}\right)\left[1+R_{1}(c)\right] \geq 1+R_{0}(c) \\
& \Rightarrow\left(1-\rho_{q}\right)\left[1+\rho_{u} \lambda+1-\rho_{u}\right] \geq 2
\end{aligned}
$$

Rearranging terms produces:

$$
\rho_{q} \leq 1-\frac{2}{2+\rho_{u}(\lambda-1)}
$$

which contradicts the conditions of proposition 1. Q.E.D.

\section{Proof of Proposition 4}

The expected payoff of an II worker who emits passing signals in both stages is

$$
1+\lambda-2 c
$$

under a C-L or L-C equilibrium. Thus, they are indifferent. If they receive a passing signal in the hiring stage and an unclear in the promotion stage, their payoff is

$$
1+\lambda-c
$$

under a C-L equilibrium and

$$
2-c
$$

under an L-C equilibrium. Thus, they strictly prefer the former.

A similar calculation will confirm that IN workers who receive a passing signal in the hiring stage and an unclear signal in the promotion stage strictly prefer a C-L equilibrium over an L-C equilibrium. And, those who receive a pass, fail are indifferent. Q.E.D. 